\title{
A commentary on "Evaluation of the histological and biomechanical properties of poly-4-hydroxybutyrate (P4HB) scaffold for pelvic organ prolapse, as compared to polypropylene mesh in a rabbit model"
}

\author{
Ghazaleh Rostaminia ${ }^{1}$ (D) \\ Received: 13 June 2021 / Accepted: 15 July 2021 / Published online: 30 July 2021 \\ (C) The International Urogynecological Association 2021
}

This prospective animal study aimed to evaluate the histological and biomechanical properties of a fully absorbable macroporous monofilament (P4HB) scaffold following abdominal and vaginal implantation in rabbit model, as compared to a lightweight polypropylene (PP) mesh. A total of 16 female (retired breeder) New Zealand White rabbits were obtained. Rabbits were randomly assigned to 3- $(n=8)$ and 9month $(\mathrm{n}=8)$ time points. P4HB scaffold used in this study had a fiber diameter of $100 \mu \mathrm{m}$, pore size of $1.5 \mathrm{~mm}^{2}$, weight of $55.4 \mathrm{~g} / \mathrm{m}^{2}$, and thickness of $280 \mu \mathrm{m}$. The lightweight PP mesh (Upsylon, Boston Scientific) used in this study had a fiber diameter of $100 \mu \mathrm{m}$, pore size of $2.8 \mathrm{~mm}^{2}$, weight of $25 \mathrm{~g} / \mathrm{m}^{2}$, and thickness of $200 \mu \mathrm{m}$. At each time point, animals were randomly assigned to either PP mesh or P4HB scaffold groups. Histological analysis: Acute inflammation, chronic inflammation, granulation tissue, fibroblast maturation, collagen deposition, neovascularization elastic fibers, and collagen deposition were scored on a scale of $0-3(0=$ none, $1=$ scant, $2=$ moderate $3=$ abundant) on H\&E-stained specimens. Biomechanical analysis: Uniaxial (load-to-failure) tensile testing was performed on the samples. A pre-load of $0.5 \mathrm{~N}$ was applied to the specimens, and the dimensions (length, width, thickness) were measured using a digital caliper. Devicetissue specimens were subjected to tensile extension at a strain rate of $0.25 \mathrm{~mm} / \mathrm{s}$ until failure, and the resultant load was recorded. Ultimate tensile strength, stiffness, and load at ultimate tensile strength were recorded.

No abdominal mesh exposures were noted. A comparable number of asymptomatic partial vaginal exposures were observed at 3 months (P4HB: $n=3$; PP: $n=2$ ) and 9 months
(P4HB: $n=3$; PP: $n=2$ ), respectively. Elastic fibers were present in the 3-month vaginal PP and P4HB specimens, but were not present at other time points or sites. Abdominally, no difference was noted; however, a trend towards an increase in the collagen I/III ratio from 3 to 9 months was observed. Biomechanical evaluation of vaginal mesh tissue complex showed ultimate tensile strength was not significantly different between P4HB and PP groups at $3(P=0.625)$ and 9 months $(P=0.250)$, respectively.

In summary, this study showed that P4HB may be biocompatible and a potential graft option for use in female pelvic organ prolapse. Both PP mesh and P4HB scaffold appear to behave similarly in the short to medium term following subcutaneous and peri-vaginal implantation in the rabbit abdomen and vagina in this small sample size. P4HB scaffold may represent a fully absorbable alternative to permanent mesh for pelvic organ prolapse (POP) repair. Knitted polyproline (PP) implants for the correction of pelvic organ prolapse have been associated with complications such as vaginal exposure, infection, and pain. There is a rationale to develop a biocompatible implant that is less prone to longterm complications. Delayed absorbable materials could meet these requirements, and poly-4-hydroxybutyrate (P4HB) might be such a new material for future pelvic floor implants.

Publisher's note Springer Nature remains neutral with regard to jurisdictional claims in published maps and institutional affiliations.

Ghazaleh Rostaminia

ghazalerostaminia@yahoo.com

1 NorthShore University HealthSystem, Skokie, IL, USA 